\title{
Structure and dynamics of two $\beta$ - peptides in solution from molecular dynamics simulations validated against experiment
}

\section{Journal Article}

Author(s):

Žagrović, Bojan; Gattin, Zrinka; Lau, Justin Kai-Chi; Huber, Matthias; van Gunsteren, Wilfred F.

Publication date:

2008-07

Permanent link:

https://doi.org/10.3929/ethz-b-000412189

Rights / license:

In Copyright - Non-Commercial Use Permitted

Originally published in:

European Biophysics Journal 37(6), https://doi.org/10.1007/s00249-008-0307-y 


\title{
Structure and dynamics of two $\beta$-peptides in solution from molecular dynamics simulations validated against experiment
}

\author{
Bojan Zagrovic · Zrinka Gattin · Justin Kai-Chi Lau • \\ Matthias Huber · Wilfred F. van Gunsteren
}

Received: 19 October 2007/Revised: 3 March 2008/Accepted: 7 March 2008/Published online: 27 March 2008 (c) EBSA 2008

\begin{abstract}
We have studied two different $\beta$-peptides in methanol using explicit solvent molecular dynamics simulations and the GROMOS 53A6 force field: a heptapeptide (peptide 1) expected to form a left-handed $3_{14}$-helix, and a hexapeptide (peptide 2) expected to form a $\beta$-hairpin in solution. Our analysis has focused on identifying and analyzing the stability of the dominant secondary structure conformations adopted by the peptides, as well as on comparing the experimental NOE distance upper bounds and ${ }^{3} \mathrm{~J}$-coupling values with their counterparts calculated on the basis of the simulated ensembles. Moreover, we have critically compared the present results with the analogous results obtained with the GROMOS 45A3 (peptide 1) and 43A1 (peptide 2) force fields. We conclude that within the limits of conformational sampling employed here, the GROMOS 53A6 force field satisfactorily reproduces experimental findings regarding the behavior of short $\beta$-peptides, with accuracy that is comparable to but not exceeding that of the previous versions of the force field.
\end{abstract}

B. Zagrovic - Z. Gattin · J. K.-C. Lau · M. Huber ·

W. F. van Gunsteren ( $\square)$

Laboratory of Physical Chemistry,

Swiss Federal Institute of Technology,

8093 Zürich, Switzerland

e-mail:wfvgn@igc.phys.chem.ethz.ch;

igc-sec@igc.phys.chem.ethz.ch

\section{B. Zagrovic}

Laboratory of Computational Biophysics,

Mediterranean Institute for Life Sciences,

Mestrovicevo setaliste bb, Split 21000, Croatia

e-mail: zagrovic@medils.hr a)
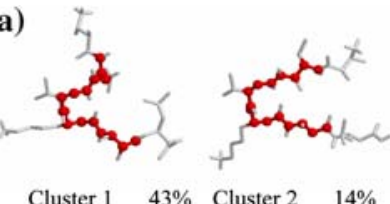

$\begin{array}{llll}\text { Cluster } 1 \quad 43 \% & \text { Cluster } 2 \quad 14 \%\end{array}$

$\mathrm{NH}(3)-\mathrm{O}(4) \quad 87 \% \quad \mathrm{NH}(2)-\mathrm{O}(5) 36 \%$

NH(4)-O(1) $35 \% \quad \mathrm{NH}(3)-\mathrm{O}(4) 71 \%$

$\mathrm{NH}(6)-\mathrm{O}(1) \quad 2 \%$ b)

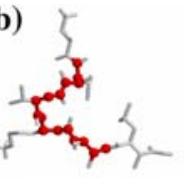

Cluster $1 \quad 50 \%$

$\mathrm{NH}(3)-\mathrm{O}(4) 87 \%$

$\mathrm{NH}(4)-\mathrm{O}(1) 33 \%$

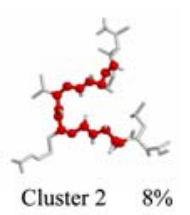

$\mathrm{NH}(3)-\mathrm{O}(4) 49 \%$

GCE legend Conformational clustering analysis of the simulated ensemble of a $\beta$-hexapeptide with two different simulation setups (a and $b$ ). The central members of all of the clusters populating more than $5 \%$ of all of the structures are shown, together with the most dominant hydrogen bonds and the corresponding percentages of cluster members containing them

Keywords GROMOS 53A6 - $\beta$-peptides . Molecular dynamics $\cdot$ Force field validation

\section{Introduction}

Foldamers are a class of non-natural polymers, which, analogous to polypeptides and nucleic acids, exhibit a strong tendency to form stable, well-defined 3-dimensional structures (Cubberley and Iverson 2001; Cheng 2004; Hecht and Huc 2007). Among the foldamers, $\beta$-peptides have recently received significant attention because of their ability to resist degradation by proteases, a feature which makes them extremely attractive for potential application as pharmaceuticals (Hintermann and Seebach 1997; Seebach et al. 1998). Moreover, $\beta$-peptides have been shown to form stable secondary structure motifs, such as helices and $\beta$-hairpins, even at sequence lengths that are much shorter than those needed for the formation of similar motifs in $\alpha$-peptides (Seebach and Matthews 1997; Cheng 
et al. 2001). As such, they are an ideal test case for studying the process of folding of secondary structure elements as well as secondary structure propensities of different $\beta$-amino acids, both in vitro and in silico.

The GROMOS 53A6 semi-empirical atomistic force field was developed with a focus on matching the thermodynamic solvation properties of amino acids, such as heats of vaporization and free energies of solvation (Oostenbrink et al. 2004, 2005). As these features of amino acids represent some of the dominant contributions to polypeptide folding (i.e., partitioning between the hydrophobic interior and the hydrophilic exterior of a typical soluble protein), it has been anticipated that a force field parameterized in such a way would be able to successfully reproduce the folding/unfolding equilibrium of a typical polypeptide. Using the GROMOS 53A6 force field, in this study we examine two different $\beta$-peptides belonging to two different structural classes: a heptapeptide (Glättli et al. 2005) (peptide 1, Fig. 1a), expected from the experiment to adopt a left-handed 314 -helical configuration, and a hexapeptide (Daura et al. 2001) (Fig. 1b, peptide 2), expected to form a $\beta$-hairpin. Peptide 1 , with the sequence $\mathrm{NH}_{3}^{+}-(S)-\beta^{3}$-HLys- $(S)-\beta^{3}$-HPhe- $(S)-\beta^{3}$-HLeu- $(S)-\beta^{3}$-HMet$(S)-\beta^{3}$-HPhe- $(S)-\beta^{3}$-HLeu- $(S)-\beta^{3}$ HLys-NH2, is an example of a $3_{14}$-helix forming $\beta$-peptide that does not contain the $\alpha$-branched side chains of Val or Ile, usually present in $31_{14}$ helical peptides. It was originally studied in methanol using $\mathrm{CD}$, NMR and molecular dynamics simulations using the GROMOS 45A3 force field. All methods pointed to a significant presence of the $33_{14}$-helix, but also suggested sizable conformational diversity as well (Glättli et al. 2005). Peptide 2 , with the sequence $\mathrm{NH}_{3}^{+}-(R, S)-\beta^{2,3}$-HAla$(R, S)$ - $\beta^{2,3}$-HVal- $(S)-\beta^{2}$-HVal- $(S)$ - $\beta^{3}$-HLys- $(R, S)-\beta^{2,3}$-HAla$(R, S)-\beta^{2,3}$-HLeu-COOH consists of two $(R, S)-\beta^{2,3}$ residues on each terminus (Fig. 1b), which promotes an extended configuration, connected by a turn-promoting $(S)-\beta^{2}-(S)-\beta^{3}$ sequence. Similar to peptide 1 , it has been studied using
$\mathrm{CD}$, NMR and MD simulations using the GROMOS 43A1 force field, all converging at the antiparallel $\beta$-hairpin configuration as the dominant structural motif adopted by the peptide in methanol (Daura et al. 2001).

For peptide 1, we carried out four different 100-ns long simulations in methanol: starting from the $33_{14}$-helix at $298 \mathrm{~K}$, we carried out simulations in the absence (simulation 1A) and in the presence (simulation 1B) of $3 \mathrm{Cl}^{-}$ counterions; second, starting from an extended structure at $340 \mathrm{~K}$ to enhance sampling, we carried out simulations in the absence (simulation 1C) and in the presence (simulation 1D) of $3 \mathrm{Cl}^{-}$counterions. For peptide 2, we carried out two 100-ns long simulations in methanol at $298 \mathrm{~K}$ : one starting from the $\beta$-hairpin structure (simulation $2 \mathrm{~A}$ ), and another from a fully extended structure (simulation 2B), with no counterions in either simulation.

The experimental structures of the two peptides were suggested on the basis of NMR experiments, and more specifically, upper distance bounds derived from Nuclear Overhauser Enhancements (NOEs) and dihedral-angle values derived from ${ }^{3} \mathrm{~J}$-coupling constants. Note that in both cases the quantity of structural information was not enough to fully constrain the structures, but it was enough to suggest the two structures as the dominant members of the ensemble. For the purposes of force-field validation, in this study we compute the values of these two key observables based on our simulated ensembles and compare the results with the experimentally measured values. In terms of the ${ }^{3} \mathrm{~J}$-coupling constants, for both peptides and in all simulations, we observe a level of agreement which exceeds that between the experimentally measured values and the values calculated for the dominant structures suggested by the experiment. In terms of the NOE-derived upper distance bounds, we note satisfactory agreement between simulation and experiment, with only a few violations. In particular, for the simulations started from the extended conformation, we observe greater violations, as a
Fig. 1 Chemical formulae of: a peptide 1 and $\mathbf{b}$ peptide 2

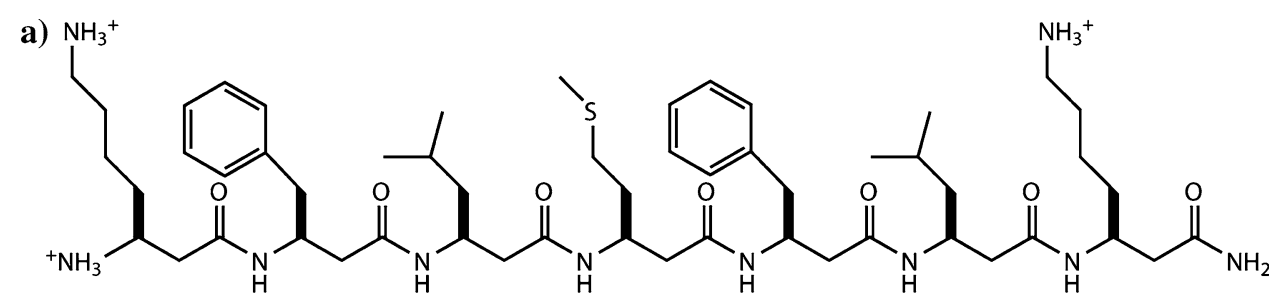

b)<smiles>CC(C)C[C@H](NC(=O)[C@H](C)[C@@H](C)NC(=O)C[C@H](CCCC[NH3+])NC(=O)[C@H](CNC(=O)[C@@H](C)[C@H](NC(=O)[C@@H](C)[C@H](C)C(=O)O)C(C)C)C(C)C)C(=O)O</smiles> 
consequence of incomplete sampling. This view is indeed corroborated by the calculated backbone atom-positional root-mean-square differences (RMSD) between the various structures and conformational clustering analysis as well. Finally, we compare the present results with the results of the simulations obtained using previous versions of the GROMOS force field: 45A3 (Schuler et al. 2001; Glättli et al. 2005) (peptide 1) and 43A1 (van Gunsteren et al. 1996; Daura et al. 2001) (peptide 2), which only differ regarding parameters for long $\left(>6 \mathrm{CH}_{2}\right.$ units) aliphatic chains, which in turn do occur in lipid membranes, but not in $\beta$-peptides. We conclude that, at the level of sampling employed here, the 53A6 force field matches in accuracy the previous versions of the GROMOS force field when it comes to capturing the behavior of short $\beta$-peptides, but does not improve on it.

\section{Materials and methods}

\section{Simulation setup}

All simulations were performed using the GROMOS simulation software package (van Gunsteren et al. 1996; Scott et al. 1999). Simulations 1A (peptide 1, no counterions), $1 \mathrm{~B}$ (peptide $1,3 \mathrm{Cl}^{-}$ions added) and $2 \mathrm{~A}$ (peptide 2) were initiated from the structures suggested by the NMR experiments; a left-handed $33_{14}$-helix for peptide 1 and a $\beta$-hairpin for peptide 2 . The initial $3{ }_{14}$-helix structure was generated by threading the sequence of peptide 1 onto the $3_{14}$-helix backbone scaffold. The initial $\beta$-hairpin structure was the lowest energy structure from the simulated annealing runs with experimental restraints [X-PLOR structure number 1 (Daura et al. 2001)]. The extended starting structures used in simulations $1 \mathrm{C}$ (peptide 1, no counterions), 1D (peptide $1,3 \mathrm{Cl}^{-}$ions added) and $2 \mathrm{~B}$ (peptide 2) were generated by setting all backbone dihedral angles to $180^{\circ}$, with the side chain dihedral angles taken randomly from the possible rotamers. For peptide 1, the $\mathrm{N}$-terminal amide group and the lysine side chains were protonated (net charge on the peptide of $+3 \mathrm{e}$ ). Note that in our simulations, unlike the NMR experiment, the C-terminus of peptide 1 was amidated. This setup parallels that in the original simulation study using the GROMOS 45A3 force field (Glättli et al. 2005), and should have no significant effect on the values of the calculated observables. For peptide 2 , the $\mathrm{N}$-terminal amide group was protonated (net charge on the peptide of $+2 \mathrm{e}$ ). All structures were placed in pre-equilibrated simulation boxes filled with methanol molecules (1A:1,373; 1B:1,370; 1C:2,144; 1D:2,141; 2A:1,490; 2B:2,494 methanol molecules). For the simulations of peptide 1 started from the helical configuration we used a rectangular box with edge sizes chosen such that the minimum distance between the peptide and the walls was $1.5 \mathrm{~nm}$. In all other cases, we used truncated octahedron boxes chosen such that the initial minimum distance between the peptide and the square walls of the box was $1.4 \mathrm{~nm}$. Simulations $1 \mathrm{~A}, 1 \mathrm{~B}, 2 \mathrm{~A}$ and 2B were carried out at $298 \mathrm{~K}$, while simulations $1 \mathrm{C}$ and $1 \mathrm{D}$ were carried out at $340 \mathrm{~K}$. In all of the simulations, after a steepest descent energy minimization, an equilibration scheme was carried out, which included gradually raising the simulation temperature from $60 \mathrm{~K}$ to the final simulation temperature, while simultaneously decreasing the atom-positional restraint coupling constant from $25,000 \mathrm{~kJ} \mathrm{~mol}^{-1}$ to $0 \mathrm{~kJ} \mathrm{~mol}^{-1}$ in equidistant steps. At each equilibration step, a short (peptide 1, 20 ps; peptide 2, $100 \mathrm{ps)}$ simulation at constant volume was carried out. This was followed by another 20 ps (peptide 1) or 100 ps (peptide 2) at the final simulation temperature and 1 atm pressure, and a subsequent production run of $100 \mathrm{~ns}$ in all six cases. Constant temperature and pressure were maintained by the Berendsen thermostat (coupling time of $0.1 \mathrm{ps}$ ) and barostat (coupling time $0.5 \mathrm{ps)}$ (Berendsen et al. 1984), respectively, using an isothermal compressibility of $4.575 \times 10^{-4}\left(\mathrm{~kJ} \mathrm{~mol}^{-1} \mathrm{~nm}^{-3}\right)^{-1}$. All simulations were carried out using the GROMOS 53A6 force field (Oostenbrink et al. 2004) under periodic boundary conditions. Electrostatics were treated using the reaction-field approach and the triple-range cutoff scheme, with cutoffs of 0.8 and $1.4 \mathrm{~nm}$, and a dielectric permittivity of 17.1, corresponding to methanol. The pair list was updated every five steps. The equations of motion were integrated using the leap-frog scheme and a step-size of 2 fs. All bonds were constrained using the SHAKE algorithm with a tolerance of 0.0001 (Ryckaert et al. 1977). Initial velocities were taken from the Maxwell-Boltzmann distribution at a given temperature.

Note that setups $1 \mathrm{~A}, 1 \mathrm{~B}, 1 \mathrm{C}$ and $1 \mathrm{D}$ were previously used in simulations with the $45 \mathrm{~A} 3$ force field (1A, one trajectory of $100 \mathrm{~ns} ; 1 \mathrm{~B}$, one trajectory of $250 \mathrm{~ns} ; 1 \mathrm{C}$, one trajectory of $200 \mathrm{~ns} ; 1 \mathrm{D}$, one trajectory of $150 \mathrm{~ns}$ ) under the same conditions, except the setup 1D, which was previously used at $298 \mathrm{~K}$ (Glättli et al. 2005). Furthermore, the setup 2B was previously simulated using the 43A1 force field (one trajectory of $100 \mathrm{~ns}$ ) (Daura et al. 2001). No simulations starting from the hairpin structure were carried out before.

\section{Analysis}

Backbone atom-positional RMSD were calculated after translational superposition of centers of mass and leastsquares rotational fitting of atomic positions, using all backbone atoms of residues 2-7 for peptide 1 , and 2-6 for peptide 2 . 
Interproton distances extracted from the NOE intensities measured in the experiment were compared with the average interproton distances in the simulations calculated using $\left\langle r^{-6}\right\rangle^{-1 / 6}$ averaging of the instantaneous interproton distances $r$. Since GROMOS force fields use the unitedatom formalism, when calculating interproton distances and averages from the simulations and comparing with the experimentally derived NOE upper distance bounds, we took the following approach (van Gunsteren et al. 1996). In the case a hydrogen atom of interest was not represented explicitly in the simulation, then either: a) a virtual atom was constructed $\left(\mathrm{CH}, \mathrm{CH}_{2}\right)$ based on standard geometries, or b) a pseudo atom was constructed and a standard pseudo atom correction was applied to the NOE upper bound (cases where NOE upper bounds were assigned to more than one proton). For a non-stereospecifically assigned $\mathrm{CH}_{2}$ group, a pseudo atom correction of $0.09 \mathrm{~nm}$ was added to the upper bound. For methyl groups, the correction was $0.1 \mathrm{~nm}$. For the methyl protons in an iso-propyl group, a correction of $0.22 \mathrm{~nm}$ was applied, and for non-assigned $\mathrm{H} \delta$ and $\mathrm{H} \varepsilon$ atoms in a flipping benzene ring, $0.21 \mathrm{~nm}$. The corrections that we used were derived based on the standard bond lengths and angles in GROMOS. We did not apply additional multiplicity corrections to the NOE upper bounds.

The ${ }^{3}$ J-coupling constants were determined using the Karplus relation (Karplus 1959):

${ }^{3} J(\mathrm{H}, \mathrm{H})=A \cos ^{2} \theta+B \cos \theta+C$

where $A=6.4 \mathrm{~Hz}, \quad B=-1.4 \mathrm{~Hz}, \quad C=1.9 \mathrm{~Hz}$ and $\theta=\phi-60^{\circ}$ for the calculation of ${ }^{3} J(\mathrm{NH}, \mathrm{CH})$ values (Pardi et al. 1984), and $A=9.5 \mathrm{~Hz}, B=-1.6 \mathrm{~Hz}$ and $C=1.8 \mathrm{~Hz}$ for the calculation of ${ }^{3} J(\mathrm{CH}, \mathrm{CH})$ values (DeMarco et al. 1978).

Conformational clustering was performed using the approach of Daura using the backbone atom-positional RMSD as the distance metric (Daura et al. 1999). A maximum cluster radius was set to $\mathrm{RMSD}=0.1 \mathrm{~nm}$ (peptide 1) or $0.08 \mathrm{~nm}$ (peptide 2), corresponding to the original studies of the peptides (Daura et al. 2001; Glättli et al. 2005). Hydrogen bonds were defined by a minimum donor-hydrogen-acceptor angle of $135^{\circ}$ and a maximum hydrogen-acceptor distance of $0.25 \mathrm{~nm}$.

\section{Results}

In Fig. 2 we analyze the backbone atom-positional RMSD from the left-handed 314 -helix (peptide 1) and $\beta$-hairpin (peptide 2) for the two simulation sets. Simulations started from the $33_{14}$-helical structure of peptide 1 , both in the absence and in the presence of counterions (Fig. 1a, curves $1 \mathrm{~A}$ and 1B) exhibit significant stability and marginal
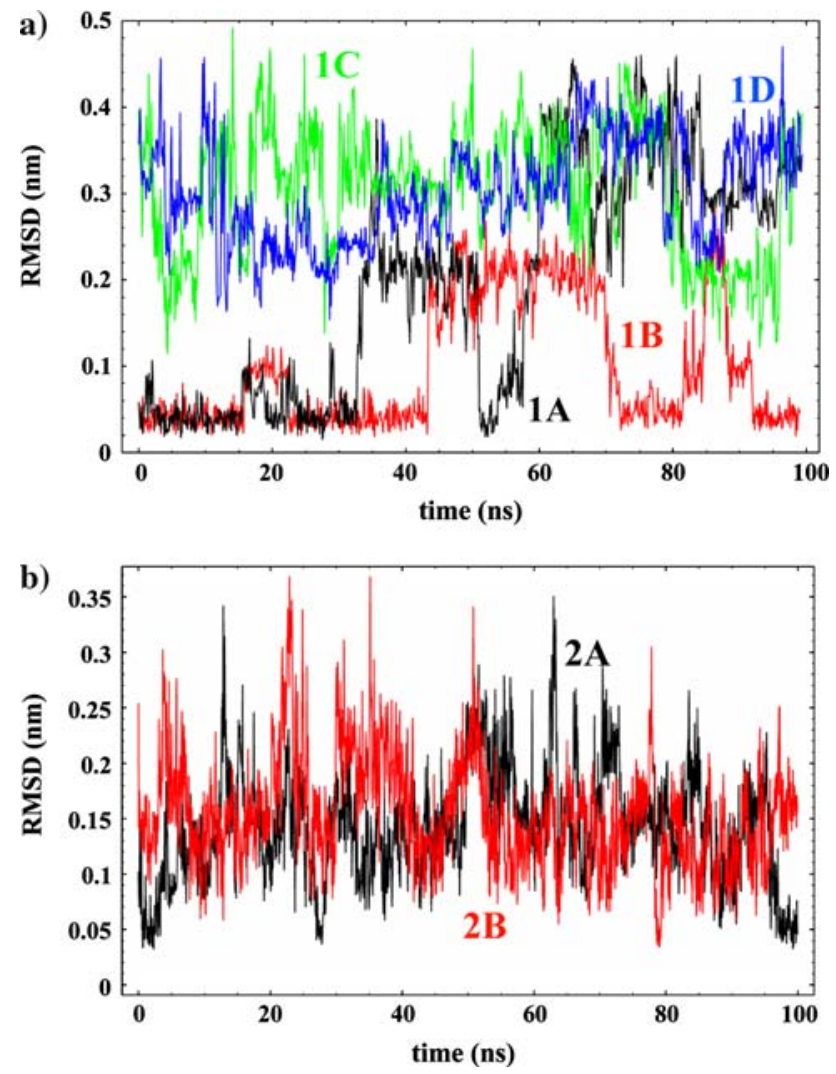

Fig. 2 a Backbone atom-positional RMSD from the 314 -helix (peptide 1) and $\mathbf{b}$ the $\beta$-hairpin (peptide 2) for different simulations. Starting from a 314 -helix at $298 \mathrm{~K}(1 \mathrm{~A}, 1 \mathrm{~B})$, from an extended structure $(1 C, 1 D)$ at $340 \mathrm{~K}$, and at $298 \mathrm{~K}$ from a $\beta$-haripin $(2 A)$ and from an extended structure $(2 B)$, without $(1 A, 1 B, 2 A, 2 B)$ and with $(1 B, 1 D)$ counterions

deviations from the experimental structure for the first 3040 ns. After about 33 ns, the simulation 1A exhibits a partial unfolding event. Visual inspection of the trajectory reveals that at this point the $\mathrm{N}$-terminal turn of the $3_{14}$-helix unfolds, resulting in an increased RMSD, while the rest of the helix remains stable. About 20 ns later, the complete helix re-forms, only to completely unfold $10 \mathrm{~ns}$ later. At this point, only the very C-terminal turn of the $3_{14}$-helix remains stable. In the presence of counterions (1B), the helix exhibits two partial unfolding events (between 43 and 72 ns, and between 83 and 93 ns) both of which result in the subsequent re-forming of the complete $33_{14}$-helix. The simulations of peptide 1 started from an extended structure (Fig. 1a, curves 1C and 1D) do not result in formation of the $34_{14}$-helix within the simulated time period, as demonstrated by the relatively large value of the RMSD throughout. Both in the absence and in the presence of counterions, these simulations result in partial folding through the formation of the C-terminal helical turn, but without ever reaching a complete $3_{14}$-helical fold. This is further discussed below in the context of the clustering analysis. 
The simulations of peptide 2 result in somewhat similar results. The trajectory started from the experimental $\beta$ hairpin structure remains fairly stable throughout, with the average atom-positional backbone RMSD from the NMR model structure of about $0.15 \mathrm{~nm}$. The simulation started from the extended conformation exhibits rapid collapse and quick formation of the proper $\beta$-hairpin turn comprising residues 3 and 4 . As a consequence, the RMSD from the complete $\beta$-hairpin rapidly reaches a relatively low level, dropping below $0.2 \mathrm{~nm}$ for a sizable fraction of time. Overall, one sees no major difference between the two simulations just on the basis of the RMSD time series, both when it comes to the average value as well as fluctuations around the average. However, differences do become apparent upon conformational clustering analysis as discussed below.

How do the experimental NMR observables compare with the values calculated on the basis of the simulated trajectories? In Fig. 3 and Table 1, we compare the ${ }^{3} \mathrm{~J}$-values calculated for the simulations of peptide 1 with their experimental counterparts. There are two notable trends: first, for both the simulations started from the folded conformation and the simulations started from the extended conformation, the average RMSD from the experimental values is somewhat lower for the simulations carried out in the absence of counterions $[0.8 \mathrm{~Hz}$ (1A) and $1.2 \mathrm{~Hz}$ (1C) vs. 1.1 (1B) and $1.6 \mathrm{~Hz}$ (1D)]. Second, the simulations started from an extended structure, which was based on the RMSD criterion never fully reach the folded conformation, exhibit only marginally greater deviations from the experimental values compared to the simulations started from the 314 -helical structure. In other words, the ${ }^{3} \mathrm{~J}$-coupling values do not appear to be overly sensitive to the overall secondary structure of the peptide. In Fig. 4, we compare the ${ }^{3} \mathrm{~J}$ values calculated for the simulations of peptide 2 with experimental values. As with peptide 1, the simulation started from the experimental structure results only in marginally better agreement with experiment compared to the simulation started from the extended structure (average RMSD of $1.1 \mathrm{~Hz}$ vs. $1.3 \mathrm{~Hz}$, respectively). As a control, it is informative to ask what the RMSD is between the experimentally measured ${ }^{3} \mathrm{~J}$-coupling values and the values calculated for the structures deemed to be most representative on the basis of the experimental data ( $3_{14}$-helix for peptide 1 and $\beta$-hairpin for peptide 2 ). Interestingly, these deviations are $1.7 \mathrm{~Hz}$ (peptide 1) and $3.6 \mathrm{~Hz}$ (peptide 2), suggesting that the ensembles provided by the simulations give a significantly better description of the microscopic reality than do the individual structures refined under the "single-structure" assumption. Curiously, this is true even for mutually very different ensembles (e.g., 1A and 1C).
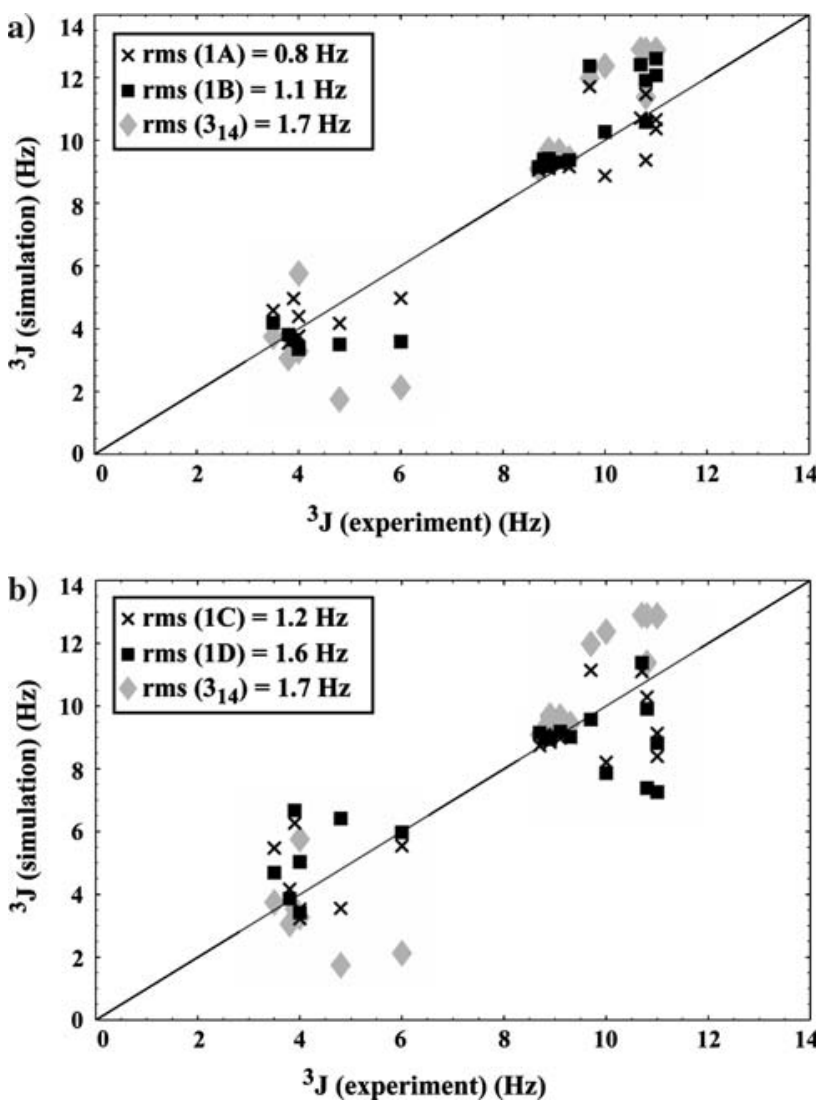

Fig. 3 Comparison of the experimentally measured ${ }^{3} \mathrm{~J}$-coupling constants (6 values for ${ }^{3} \mathrm{~J}_{\mathrm{HN}-\mathrm{H} \beta}$ and 14 values for ${ }^{3} \mathrm{~J}_{\alpha \beta}$-coupling constants) with the corresponding ${ }^{3} \mathrm{~J}$-couplings calculated based on the simulated trajectories for peptide 1: a simulation 1A (crosses) and 1B (boxes), and b simulation 1C (crosses) and 1D (boxes). Rootmean-square deviations from the experimental values are denoted with rms. In both $\mathbf{a}$ and $\mathbf{b}$ the comparison between the experimental values and the ideal 314 -helix is depicted by gray diamonds. Experimental data were obtained at $298 \mathrm{~K}$, while simulations were carried out at $298 \mathrm{~K}(1 \mathrm{~A}, 1 \mathrm{~B})$ and $340 \mathrm{~K}(1 \mathrm{C}, 1 \mathrm{D})$

The experimental characterization of both peptides was largely based on the NOE-derived upper distance bounds. How do these values compare with the ones calculated from our simulations? In Fig. 5 and Table 1, we analyze the upper distance bound violations for the simulations of peptide 1 . The simulated ensembles violate the experimental upper distance bounds for a handful of proton pairs only, with the average violations overall of the experimentally measured NOEs ranging from $0.005 \mathrm{~nm}$ in the case of the simulation $1 \mathrm{~A}$ to $0.014 \mathrm{~nm}$ in the case of the simulation 1C. As expected, the violations are greater for the simulations started from the extended conformation, as these never reached the completely folded conformation. However, it is remarkable that even still the average violations for these simulations are fairly low. This can be attributed to the fact that the majority of the measured NOEs do not report on the global, long-range structure of 
Table 1 Comparison between the present simulations using the GROMOS 53A6 force field and the previous simulations using force fields $45 \mathrm{~A} 3$ (peptide 1) and 43A1 (peptide 2) with the number of chloride ions, temperature, starting structure (ext. stands for extended structure) and simulation length as given

\begin{tabular}{|c|c|c|c|c|c|c|c|c|c|c|c|c|}
\hline \multirow{3}{*}{$\begin{array}{l}\text { Molecule } \\
\text { Setup } \\
\text { Force field }\end{array}$} & \multicolumn{8}{|c|}{ Peptide 1} & \multicolumn{4}{|c|}{ Peptide 2} \\
\hline & \multicolumn{2}{|l|}{$1 \mathrm{~A}$} & \multicolumn{2}{|l|}{$1 \mathrm{~B}$} & \multicolumn{2}{|l|}{$1 \mathrm{C}$} & \multicolumn{2}{|l|}{$1 \mathrm{D}$} & \multicolumn{2}{|l|}{$2 \mathrm{~A}$} & \multicolumn{2}{|l|}{$2 \mathrm{~B}$} \\
\hline & $53 \mathrm{~A} 6$ & $45 \mathrm{~A} 3$ & $53 \mathrm{~A} 6$ & $45 \mathrm{~A} 3$ & $53 \mathrm{~A} 6$ & $45 \mathrm{~A} 3$ & $53 \mathrm{~A} 6$ & $45 \mathrm{~A} 3$ & $53 \mathrm{~A} 6$ & $43 \mathrm{~A} 1$ & $53 \mathrm{~A} 6$ & $43 \mathrm{~A} 1$ \\
\hline Number of $\mathrm{Cl}^{-}$ions & 0 & 0 & 3 & 3 & 0 & 0 & 3 & 3 & 0 & - & 0 & 0 \\
\hline $\mathrm{T}(\mathrm{K})$ & 298 & 298 & 298 & 298 & 340 & 340 & 340 & 298 & 298 & - & 298 & 298 \\
\hline Initial & Helix & Helix & Helix & Helix & Ext. & Ext. & Ext. & Ext. & Hairpin & - & Ext. & Ext. \\
\hline Length (ns) & 100 & 100 & 100 & 250 & 100 & 200 & 100 & 150 & 100 & - & 100 & 100 \\
\hline$<\left(\Delta^{3} \mathrm{~J}\right)^{2}>^{1 / 2}(\mathrm{~Hz})$ & 0.8 & 1.2 & 1.1 & 0.8 & 1.2 & 1.0 & 1.6 & 1.2 & 1.1 & - & 1.3 & 2.2 \\
\hline <viol_noe> (nm) & 0.005 & 0.002 & 0.006 & 0.003 & 0.014 & 0.010 & 0.013 & 0.009 & 0.001 & - & 0.015 & 0.021 \\
\hline \# Clusters & 109 & 195 & 11 & 323 & 328 & 668 & 217 & 213 & 96 & - & 109 & 197 \\
\hline
\end{tabular}

Root-mean-square deviations from the experimental values for the ${ }^{3} \mathrm{~J}$-coupling constants from the simulations were calculated over all ${ }^{3} \mathrm{~J}$ coupling constants reported from experiments at $298 \mathrm{~K}$ (peptide 1,6 values for ${ }^{3} \mathrm{~J}_{\mathrm{HN}-\mathrm{H} \beta}$ and 14 values for ${ }^{3} \mathrm{~J}_{\alpha \beta}$-coupling constants; peptide 2 , 6 values for ${ }^{3} \mathrm{~J}_{\mathrm{HN}-\mathrm{H} \beta}$ and 8 values for ${ }^{3} \mathrm{~J}_{\alpha \beta}$-coupling constants). Similarly, the average violations of the NOE upper distance bounds were calculated over all NOEs (peptide 1,84; peptide 2,20) reported in the experimental study. The total number of conformational clusters in simulations is denoted by \# clusters

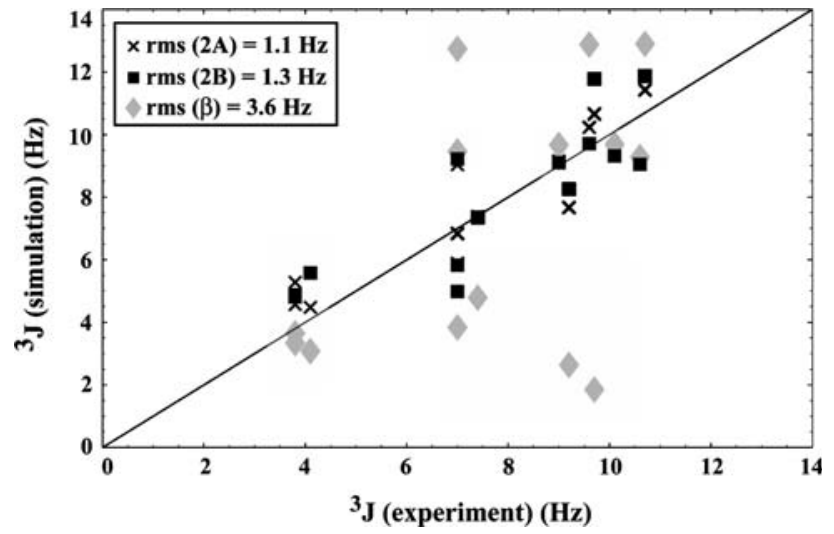

Fig. 4 Comparison of the experimentally measured ${ }^{3} \mathrm{~J}$-coupling constants (6 values for ${ }^{3} \mathrm{~J}_{\mathrm{HN}-\mathrm{H} \beta}$ and 8 values for ${ }^{3} \mathrm{~J}_{\alpha \beta}$-coupling constants) with the corresponding ${ }^{3} \mathrm{~J}$-couplings calculated based on the simulated trajectories for peptide 2: simulation $2 \mathrm{~A}$ (crosses) and 2B (boxes). Root-mean-square deviations from the experimental values are denoted with rms. The comparison between the experimental values and the ideal $\beta$-hairpin is depicted by gray diamonds. Simulations were run at the same temperature $(298 \mathrm{~K})$ at which the experimental data were obtained

the peptide, but rather on the short-sequence-range distances, which are not sensitive to it. In Fig. 6, we show the equivalent results for the two simulations of peptide 2 . For the simulation started from the hairpin structure, there is only one upper-distance bound violation of only $0.002 \mathrm{~nm}$. For the simulation started from the extended structure, there are two violations, one of which is significant $(0.25 \mathrm{~nm})$. The pair of protons involved is located at the open ends of the hairpin (HA in residue 1 and HB in residue 6) and as the simulation $2 \mathrm{~B}$ never results in a fully formed hairpin, this distance bound is violated. Overall, when it comes to ${ }^{3} \mathrm{~J}$-coupling values and the NOE-derived upper distance bounds, the present simulations (53A6) of peptide 1 agree less well with experiment compared to the results obtained with the $45 \mathrm{~A} 3$ version of the force field (Table 1), whereas for peptide 2 the opposite is observed with respect to the 43A1 simulations.

In order to get a sense of the dominant structures populated in our simulations, we have carried out a conformational clustering analysis, the results of which are shown in Fig. 7 and Table 1 for peptide 1. As expected, the dominant conformation for the simulations $1 \mathrm{~A}$ and $1 \mathrm{~B}$ is indeed the experimentally observed left-handed 3 -helix (Fig. 7a, b). Both simulations 1A and 1B (Fig. 7a, b) exhibit partial unfolding, and, consequently, the second most populated cluster for both simulations (16 and $27 \%$ of all structures, for $1 \mathrm{~A}$ and $1 \mathrm{~B}$, respectively) contains structures with a frayed $\mathrm{N}$-terminus and a 314 -helical Cterminus, stabilized by backbone hydrogen bonds between residues 4 and 6 , and 5 and 7 . The simulations started from an extended conformation (1C and 1D) exhibit a significantly greater structural diversity, which is reflected in the fact that the most numerous clusters encompass only 20 and $13 \%$ of all structures, respectively. In the absence of counterions (1C, Fig. 7c), one observes a significant dominance of structures exhibiting partial formation of the $3_{14}$-helical conformation at the C-terminus as evident from the formation of the corresponding hydrogen bonds. No such helix formation is seen in the presence of counterions.

A similar clustering analysis was carried out for peptide 2 as well (Fig. 8 and Table 1). As expected, for the simulations started from the $\beta$-hairpin structure, the two most dominant clusters (accounting together for $57 \%$ of all of the structures) center around hairpin-like conformations. The most numerous cluster ( $43 \%$ of all structures) includes 

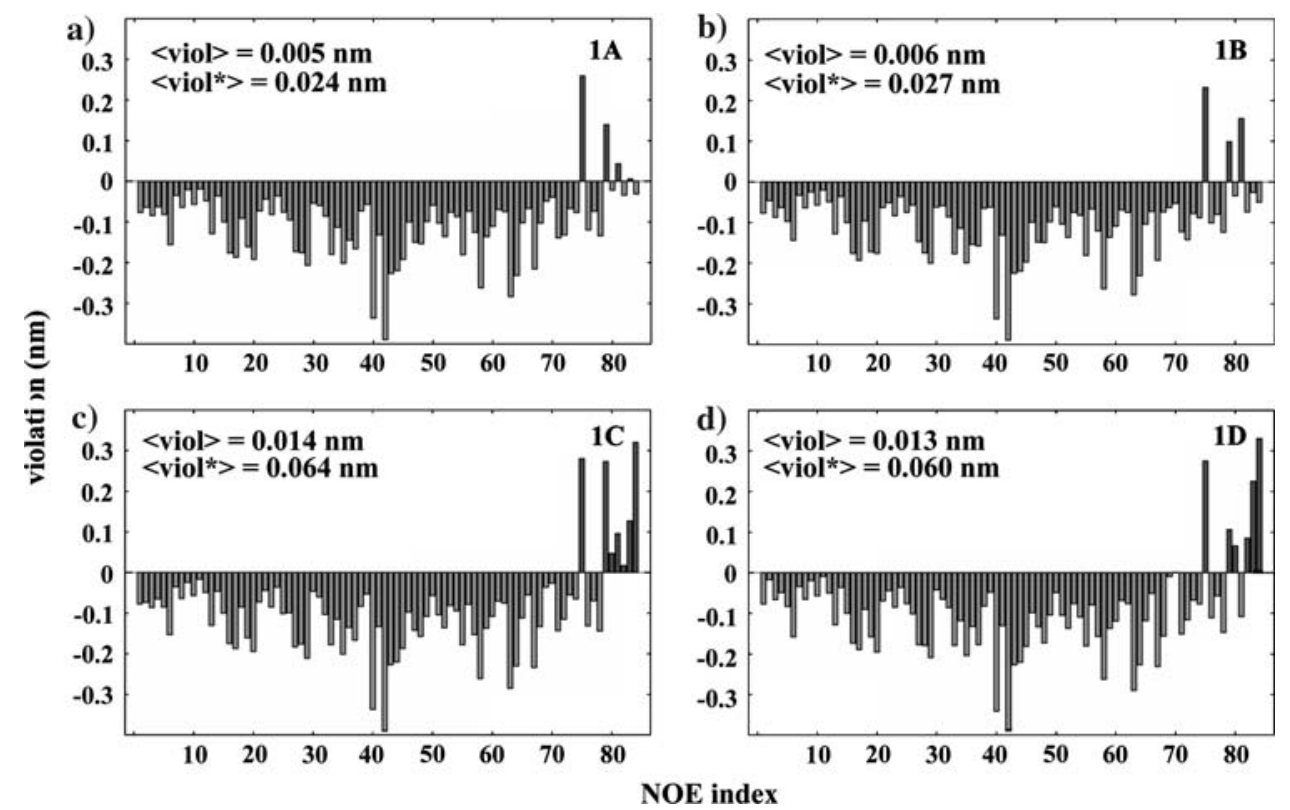

Fig. 5 Violations of the experimentally derived upper distance bounds for the simulated trajectories of peptide 1: a simulation $1 A$, b simulation $1 B$, c simulation $1 C$ and $\mathbf{d}$ simulation $1 D$. Negative violations (light bars) indicate cases where the ensemble average distance from simulations is less than the experimental upper distance bound. Average violations calculated over the entire set of upper

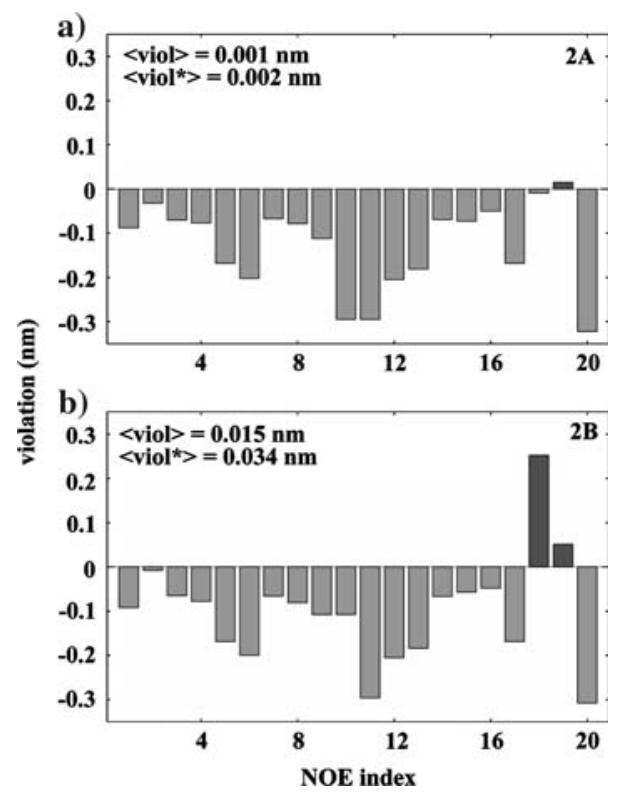

Fig. 6 Violations of the experimentally derived upper distance bounds for the simulated trajectories of peptide 2: a simulation $2 \mathrm{~A}$ and $\mathbf{b}$ simulation $2 B$. Negative violations (light bars) indicate cases where the ensemble average distance from simulations is less than the experimental upper distance bound. Average violations calculated over the entire set of upper distance bounds (20 values), $<$ viol $>$, and average violations calculated only over inter-residue upper distance bounds ( 9 values), $\left\langle\right.$ viol $\left.^{*}\right\rangle$, are also given. Simulations were run at the same temperature $(298 \mathrm{~K})$ at which the experimental data were obtained distance bounds (84 values), <viol $>$, and average violations calculated only over inter-residue upper distance bounds (18 values), $\left\langle\right.$ viol $^{*}>$, are also given. Experimental data were obtained at $298 \mathrm{~K}$, while simulations were carried out at $298 \mathrm{~K}(1 \mathrm{~A}, 1 B)$ and $340 \mathrm{~K}(1 C$, 1D)

$\beta$-hairpin conformations with both ends frayed, while the second-most numerous cluster (14\%) centers around the fully formed $\beta$-hairpin (Fig. 8a). In both cases, the hairpin turn is stabilized by persistent backbone-backbone hydrogen bonds between residues 3 and 4 . In terms of the overall behavior, the hairpin apparently unfolds by "unzipping" of the hydrogen bonds from the termini in the direction of the turn; in the majority of structures this unzipping includes the two hydrogen bonds bridging the chain termini (cluster 1 with $43 \%$ of all structures).

As already hinted in the above backbone atom-positional RMSD analysis, the most dominant cluster of peptide 2 in the simulations started from the extended structure (cluster 1 , accounting for $50 \%$ of the entire ensemble, Fig. 8b) includes structures with a fully formed turn and persistent 34 and 4-1 hydrogen bonds, but with two residues on either end frayed (analogous to the first cluster in the simulations started from the folded conformation). The two other most populated clusters for this simulation contain somewhat more irregular, varying structures, with no persistent hydrogen bonds, and no hairpin turn formed.

\section{Discussion}

An important difference between the simulations of peptides 1 and 2 using the 45A3 (Glättli et al. 2005) and 43A1 (Daura et al. 2001) force fields, as opposed to the 53A6 
a)

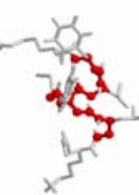

Cluster $1 \quad 39 \%$

$\mathrm{NH}(2)-\mathrm{O}(4) 94 \%$ $\mathrm{NH}(3)-\mathrm{O}(5) 94 \%$ $\mathrm{NH}(4)-\mathrm{O}(6) 68 \%$ NH(5)-O(7) $64 \%$

c)

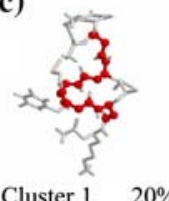

$\mathrm{NH}(4)-\mathrm{O}(6) 89 \%$ NH(5)-O(7) $78 \%$

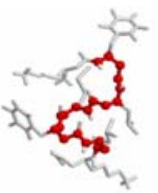

Cluster $2 \quad 16 \%$ $\mathrm{NH}(2)-\mathrm{O}(3) 9 \%$ $\mathrm{NH}(4)-\mathrm{O}(6) 93 \%$ $\mathrm{NH}(5)-\mathrm{O}(7) 79 \%$

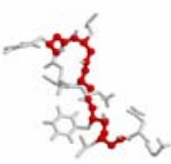

Cluster $2 \quad 11 \%$ $\mathrm{NH}(2)-\mathrm{O}(3) 3 \%$ b)

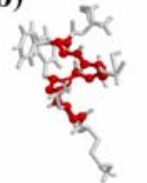

Cluster $1 \quad 66 \%$ Cluster $2 \quad 27 \%$ $\mathrm{NH}(2)-\mathrm{O}(4) 87 \% \quad \mathrm{NH}(4)-\mathrm{O}(6) 94 \%$ $\mathrm{NH}(3)-\mathrm{O}(5) 96 \% \quad \mathrm{NH}(5)-\mathrm{O}(7) 91 \%$ $\mathrm{NH}(4)-\mathrm{O}(6) 85 \%$ $\mathrm{NH}(5)-\mathrm{O}(7) 71 \%$

d)

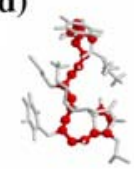

Cluster $1 \quad 13 \% \quad$ Cluster $2 \quad 11 \%$ NH(7)-O(4) $43 \% \quad$ NH(2)-O(6) $21 \%$ $\mathrm{NH}(7)-\mathrm{O}(5) 17 \% \quad \mathrm{NH}(2)-\mathrm{O}(7) 44 \%$ $\mathrm{NH}(3)-\mathrm{O}(4) 57 \%$

Fig. 7 Conformational clustering analysis of the simulated ensembles of peptide 1 for: a simulation 1A $(298 \mathrm{~K})$, b simulation 1B $(298 \mathrm{~K})$, c simulation 1C (340 K) and d simulation 1D (340 K). The central members of all of the clusters populating more than $5 \%$ of all the structures are shown, together with the most dominant hydrogen bonds and the corresponding percentages of cluster members containing them

a)

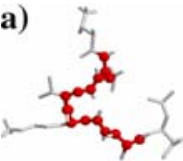

Cluster $1 \quad 43 \%$ Cluster $2 \quad 14 \%$

NH(3)-O(4) $87 \% \quad$ NH(2)-O(5) $36 \%$

$\mathrm{NH}(4)-\mathrm{O}(1) 35 \% \quad \mathrm{NH}(3)-\mathrm{O}(4) 71 \%$ $\mathrm{NH}(6)-\mathrm{O}(1) \quad 2 \%$ b)

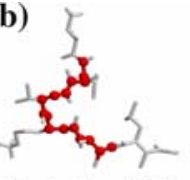

Cluster $1 \quad 50 \%$

NH(3)-O(4) $87 \%$

$\mathrm{NH}(4)-\mathrm{O}(1) 33 \%$

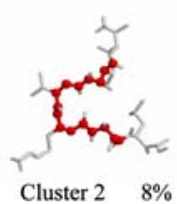

NH(3)-O(4) $49 \%$

Fig. 8 Conformational clustering analysis of the simulated ensemble of peptide 2 for: a simulation 2A and b simulation 2B, both at $298 \mathrm{~K}$. The central members of all the clusters populating more than $5 \%$ of all the structures are shown, together with the most dominant hydrogen bonds and the corresponding percentages of cluster members containing them

force field, is that the simulations using the former resulted in a significantly greater conformational diversity of structures, i.e., a much larger number of comparatively less populated conformational clusters. For example, the largest cluster in setup 1A accounts for only $11 \%$ of all structures with $45 \mathrm{~A} 3$, while in the case of 53A6 the largest cluster includes $39 \%$ of all structures. Together, the two largest clusters of $1 \mathrm{~A}$ with $45 \mathrm{~A} 3$ account for only $19 \%$ of all structures, while in the case of 53A6 this fraction is $55 \%$. Similarly, in the case of setup 1B, the two largest clusters account for $51 \%$ of all conformations with $45 \mathrm{~A} 3$, while with $53 \mathrm{~A} 6$ this number is $93 \%$. Finally, the situation is repeated with setup 1C (45A3:14\% vs. $53 \mathrm{~A} 6: 31 \%$ of all structures in the two largest clusters). In $1 \mathrm{D}$, the situation is

seemingly reversed $(45 \mathrm{~A} 3: 30 \%$ vs. $53 \mathrm{~A} 6: 24 \%$ of all structures in the two largest clusters). However, one should remember that the simulations of 1D with 53A6 were carried out at $340 \mathrm{~K}$, while the ones with $45 \mathrm{~A} 3$ were carried at out $298 \mathrm{~K}$. The difference between the $45 \mathrm{~A} 3$ and the $53 \mathrm{~A} 6$ force field reflects itself in the total number of clusters as well (Table 1), except in 1D. Note again that in this case the original simulation with $45 \mathrm{~A} 3$ was carried out at $298 \mathrm{~K}$, which should by itself result in a smaller number of clusters. When it comes to simulations of peptide 2, the situation is similar. While, for example, the total number of clusters in simulation $2 \mathrm{~B}$ with the $53 \mathrm{~A} 6$ force field is 109 , the equivalent number in the case of the $43 \mathrm{~A} 1$ simulations was 197, for the same length of simulation (Table 1). In the case of peptide 1, a part of the explanation probably lies in the, on average, lower level of sampling in the present study when it comes to simulations 1B [250 ns in Glättli et al. (2005) vs. $100 \mathrm{~ns}$ here] and 1C [200 ns in Glättli et al. (2005) vs. $100 \mathrm{~ns}$ here]. However, this difference is present in simulations 1A, 2A and 2B as well, where the simulation lengths were the same for different force fields. Overall, it appears that the 53A6 force field simply results in less variety of structures. A potential explanation lies in the comparatively larger intramolecular electrostatic interactions in the case of the 53A6 force field, most notably stronger hydrogen bonds resulting from the increased partial charges. These interactions trap and fix the molecule in a given configuration for longer periods of time in the case of the 53A6 force field, resulting in slower coverage of the phase space compared to when using previous force fields. The low dielectric permittivity of methanol only potentiates the importance of such interactions, in comparison to, say, aqueous environments. Here, it should also be mentioned that in the case of the 53A6 force field, no formation of the complete $34_{14}$-helix is observed in the simulated $100 \mathrm{~ns}$ starting from the extended conformation, while in the analogous situation with $45 \mathrm{~A} 3$ within the same amount of time (the first $100 \mathrm{~ns}$ ) multiple folding-unfolding events are observed (a total of five). It is likely that this difference can be traced back to the fact that, as discussed here, the simulations with the 45A3 force field explore the configuration space more quickly.

In the original simulations of peptide 1 using the GROMOS 45A3 force field, a small but noticeable population of right-handed $2.5_{12}$-helices was observed. These helices, which are experimentally observed only with $\beta$-peptides containing mainly trans-2-aminocyclopentanecarboxylic acid (trans-ACPC), are characterized by 12membered hydrogen bonded rings (NH(i)-O(i - 3)) (Appella et al. 1997, 1999). However, in the present study this motif is observed only in a small subset of structures in the 1D simulation (cluster 1,13\% of all structures). Unlike the $45 \mathrm{~A} 3$ simulations where there was an instance of a 
complete, albeit temporary $2.5_{12}$-helix formation, no such event was observed in the present simulations. One of the principal differences between the $45 \mathrm{~A} 3$ and $53 \mathrm{~A} 6$ force fields are the increased partial charges on the backbone carbonyls and amides in the latter (Oostenbrink et al. 2004). It is possible that this results in effectively stronger backbone hydrogen bonds in 53A6. As the formation of $3_{14}$ hydrogen bonds occurs more readily due to more favorable torsional angles, it is possible that stronger hydrogen bonds in the case of the 53A6 force field fix and stabilize these helical structures for longer periods of time relative to the $45 \mathrm{~A} 3$ case, resulting in their predominance over $2.5_{12}$ structures. However, it should be stressed that both force fields agree qualitatively in that the $3_{14}$-helices are significantly more preferred.

When it comes to the effect of counterions, the present simulations of peptide 1 parallel qualitatively the results using the 45A3 force field (Glättli et al. 2005), namely, for the simulations started from the $3_{14}$-helix in both cases it is observed that the presence of counterions stabilizes the helix, while for the simulations started from an extended structure in both cases no such marked difference is seen in the presence of counterions. However, as in the original $45 \mathrm{~A} 3$ study, no clear correlation is observed between the ion-peptide distances and the stability of the $33_{14}$-helical conformation (data not shown). Overall, it appears that the effect of counterions on the stability of peptides cannot easily be explained on the basis of the simulations presented here, and it is possible that the differences seen for the simulations started from the $3_{14}$-helix in both studies are not statistically significant. For a complete and exhaustive treatment, one would need to simulate an ensemble of trajectories with different initial conditions, which was beyond the scope of this study.

In the original simulation study of peptide 1 (Glättli et al. 2005), significant attention was given to the analysis of the interactions between the charged $\beta$-lysine residues and the backbone carbonyls, and it was suggested that these interactions compete with the backbone hydrogen bonds and disrupt the $3_{14}$-helix. A similar analysis was carried out here as well (data not shown). In general, the positively charged $\beta$-lysines were indeed found to interact with the backbone carbonyls, and these interactions were, as in the original study, seen to be affected by the presence of counterions; namely, for the simulations started from the helical structure in the presence of counterions (1B), there were no such interactions seen. However, it is possible that this is simply a secondary consequence of the fact that only in $1 \mathrm{~B}$, the $3_{14}$-helix remained more or less intact over time, while the other simulations resulted in more diverse, fluctuating and structurally open ensembles, in which there was greater opportunity for such interactions. Overall, the total number of binding-unbinding events of lysine side chains to the backbone carbonyls was comparable between the two force fields (data not shown).

To summarize, within the limits of sampling employed here, the agreement between simulation and experiment in the case of the 53A6 force field is comparable to that of the previous versions of the force field, somewhat worse for peptide 1 and better for peptide 2 . The most marked difference concerning the new force field is that it results in significantly slower coverage of the phase space, i.e., lower conformational diversity of structures, for the same length of simulation. The exact reasons for this observation as well as a more rigorous comparison with experiment to determine which force field results in more realistic dynamics should be a topic for further investigation.

Acknowledgments B.Z acknowledges support from an EMBO postdoctoral fellowship. This work was financially supported by grants from the National Center of Competence in Research (NCCR) in Structural Biology of the Swiss National Science Foundation, which is gratefully acknowledged.

\section{References}

Appella DH, Christianson LA, Karle IL, Powell DR, Gellman SH (1997) Polypeptides of cyclic beta-amino acids that form stable helices. Abstr Pap Am Chem Soc 213:328-ORGN

Appella DH, Christianson LA, Karle IL, Powell DR, Gellman SH (1999) Synthesis and characterization of trans-2-aminocyclohexanecarboxylic acid oligomers: an unnatural helical secondary structure and implications for beta-peptide tertiary structure. J Am Chem Soc 121:6206-6212

Berendsen HJC, Postma JP, van Gunsteren WF, DiNola A, Haak JR (1984) Molecular dynamics with coupling to an external bath. J Chem Phys 81:3684-3690

Cheng RP (2004) Beyond de novo protein design—de novo design of non-natural folded oligomers. Curr Opin Struct Biol 14:512-520

Cheng RP, Gellman SH, DeGrado WF (2001) Beta-peptides: from structure to function. Chem Rev 101:3219-3232

Cubberley MS, Iverson BL (2001) Models of higher-order structure: foldamers and beyond. Curr Opin Chem Biol 5:650-653

Daura X, van Gunsteren WF, Mark AE (1999) Folding-unfolding thermodynamics of a beta-heptapeptide from equilibrium simulations. Proteins 34:269-280

Daura X, Gademann K, Schäfer H, Jaun B, Seebach D, van Gunsteren WF (2001) The beta-peptide hairpin in solution: conformational study of a beta-hexapeptide in methanol by NMR spectroscopy and MD simulation. J Am Chem Soc 123:2393-2404

DeMarco A, Llinas M, Wüthrich K (1978) H-1-N-15 spin-spin couplings in alumichrome. Biopolymers 17:2727-2742

Glättli A, Daura X, Bindschädler P, Jaun B, Mahajan YR, Mathad RI, Rueping M, Seebach D, van Gunsteren WF (2005) On the influence of charged side chains on the folding-unfolding equilibrium of beta-peptides: a molecular dynamics simulation study. Chemistry 11:7276-7293

Hecht E, Huc I (eds) 2007 Foldamers: structure, properties, and applications. Wiley-VCH, New York

Hintermann T, Seebach D (1997) The biological stability of beta-peptides: no interactions between alpha- and beta-peptidic structures. Chimia 51:244-247

Karplus M (1959) Contact electron-spin coupling of nuclear magnetic moments. J Chem Phys 30:11-15 
Oostenbrink C, Villa A, Mark AE, van Gunsteren WF (2004) A biomolecular force field based on the free enthalpy of hydration and solvation: the GROMOS force-field parameter sets 53A5 and 53A6. J Comput Chem 25:1656-1676

Oostenbrink C, Soares TA, van der Vegt NF, van Gunsteren WF (2005) Validation of the 53A6 GROMOS force field. Eur Biophys J 34:273-284

Pardi A, Billeter M, Wüthrich K (1984) Calibration of the angular dependence of the amide proton- $\mathrm{C}$ alpha proton coupling constants, ${ }^{3} \mathrm{~J}_{\mathrm{HN} \alpha}$, in a globular protein. Use of ${ }^{3} \mathrm{~J}_{\mathrm{HN} \alpha}$ for identification of helical secondary structure. J Mol Biol 180:741-751

Ryckaert JP, Ciccotti G, Berendsen HJC (1977) Numerical integration of Cartesian equations of motion of a system with constraintsmolecular dynamics of n-alkanes. J Comput Phys 23:327-341

Scott WRP, Hünenberger PH, Tironi IG, Mark AE, Billeter SR, Torda AE, Huber T, Krüger P, van Gunsteren WF (1999) The
GROMOS biomolecular simulation program package. J Phys Chem A 103:3596-3607

Seebach D, Matthews JL (1997) Beta-peptides: a surprise at every turn. Chem Comm21:2015-2022

Seebach D, Abele S, Schreiber JV, Martinoni B, Nussbaum AK, Schild H, Schulz H, Hennecke H, Woessner R, Bitsch F (1998) Biological and pharmacokinetic studies with beta-peptides. Chimia 52:734-739

Schuler LD, Daura X, Van Gunsteren WF (2001) An improved GROMOS96 force field for aliphatic hydrocarbons in the condensed phase. J Comput Chem 22:1205-1218

van Gunsteren WF, Billeter SR, EisingAA, Hünenberger PH, Krüger P, Mark AE, Scott WRP, Tironi IG (1996) Biomolecular simulation: the GROMOS96 manual and user guide. Biomos, Zurich, Groningen 